\title{
RELAÇOES ENTRE CARACTERISTICAS QUÍMICAS DO SOLO E CRESCIMENTO DE Araucaria angustifolia (Bert.) O. Ktze., EM PASSO FUNDO RS
}

Carmeli Antonia Cassol e Ari Zago

Departamento de Solos. Centro de Ciências Rurais. UFSM. Santa Maria, RS.

RESUMO

0 presente estudo mostra as relações entre características químicas do solo e o crescimento em povoamento de Araucaria angustifolia (Bert.) 0. Ktze., implantado em diferentes tipos de solos, na Flo resta Nacional de Passo Fundo, RS. Os solos foram classificados co mo Latosol Roxo, Solo Litōlico Eutrófico e Gley Pouco Húmico.

A amostragem dos solos foi realizada na profundidade de 0 - $20 \mathrm{~cm}$, aleatoriamente, em parcelas quadradas de $400 \mathrm{~m}^{2}$.

As características químicas dos solos foram relacionadas às variāveis do crescimento utilizando-se correlações simples linea res.

Pelos resultados obtidos, nas condições deste estudo, as características químicas do solo não foram consideradas parâmetros adequados para explicar a variação do crescimento de Araucaria angustifolia.

SUMMARY

CASSOL, C.A., and ZAGO, A., 1983. Relationships between chemical soil characteristics and growth of Araucaria angustifotia (Bert.) 0 . Ktze., in Passo Fundo, RS. Ciêneia e Natura, 5:95-101.

The present work shows chemical soil characteristics and growth in plantings of Araucaria angustifolia, implanted in diffe rent soil types, in Passo Fundo, RS National Forest. Soil types were classified as Latosol Roxo, Lithosol Eutrophic Soil and Low - Humic Gley.

Soil samples were taken randomly at $0-20$ an deep in $400 \mathrm{~m}^{2}$ area square plots.

The chemical characteristics of the studied soils were related to growth variables by simple linear correlation.

According to the findings, the chemical soil characteris tics were not considered adequate parameters to explain the growth variation observed for Araucaria angustifolia.

INTRODUÇÃO

0 crescimento de florestas, nem sempre condiz com a apa rente aptidão dos solos para espécies implantadas. Isto decorre do fato de que muitos fatores influem nas relações solo-crescimento. 0 co 
nhecimento do grau de influência, destes fatores, pode contribuir para aumentar o crescimento, e, conseqdentemente, a produtividade das espēcies.

Segundo VAN GOOR (5) a relação mais importante para o cres cimento de Araucaria angustifolia è o teor de Ca + Mg do solo. Con sidera como minimo o teor de $2 \mathrm{me} / 100 \mathrm{~g}$ de solo.

A determinação da disponibilidade de nutrientes, em solos sob florestas é difícil, uma vez que, muitos fatores estão envolvi dos, DE HOOGH (2). Alëm disso, a quantidade de nutrientes contidos na serrapilheira, restos de raízes subterrâneas e na micorriza, que ocorrem associados com Araucaria angustifolia, podem contribuir pa ra a disponibilidade de nutrientes, ANDRAE \& KRAPFENBAUER (1).

DE HOOGH \& DIETRICH (3) em estudos realizados em Passo Fundo, RS, encontraram relações significantes entre teores de potás sio, fōsforo, capacidade de troca, pH, alumīnio, cālcio mais magnē sio com o indice de sîtio. A percentagem de saturação de bases foi mais significativa e correlacionou-se positivamente com o crescimen to em altura de Araucaria angustifolia.

HOPPE (4) em Passo Fundo, RS, obteve correlações negativas entre os teores de fósforo, potássio e magnēsio nas duas profundida des de estudo $(0-30 \mathrm{~cm}$ e $0-50 \mathrm{~cm})$ e observou idêntica correla ção entre o teor de cālcio na profundidade de $0-50 \mathrm{~cm}$, com altura dominante de Araucaria angustifolia.

0 estudo das relações entre características químicas do solo e as variāveis do crescimento de Araucaria angustifolia consis tem numa investigação da influência no crescimento, pois presume-se que os nutrientes do solo, poderiam contribuir no aumento da produ tividade da espécie em estudo.

MATERIAL E METODOS

Os solos, para o presente estudo, foram classificados co mo: Latosol Roxo Húmico ālico textura argilosa, Solo Litólico Eutrō fico textura franca e Gley Pouco Húmico Eutrōfico textura argilosa.

Amostras dos solos foram coletadas em parcelas quadradas de $400 \mathrm{~m}^{2}$ na profundidade de $0-20 \mathrm{~cm}$.

As anālises quĩmicas foram feitas de acordo com os métodos descritos em VETTORI (6) e incluem: carbono orgânico, com bicromato de potássio $0,4 \mathrm{~N}$ e transformado em matēria orgânica pelo fator 1,$724 ; \mathrm{Al}^{+++}$, $\mathrm{Ca}^{++} \mathrm{e} \mathrm{Mg}^{++}$trocāveis com solução extratora $\mathrm{KCl}^{1} \mathrm{~N}$; $\mathrm{Na}^{+}$e $\mathrm{K}^{+}$trocá

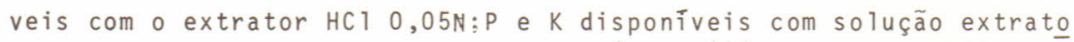
ra $0,05 \mathrm{~N}$ em HCl e $0,025 \mathrm{~N} \mathrm{em} \mathrm{H}_{2} \mathrm{SO}_{4} ; \mathrm{H}^{+}+\mathrm{Al}^{+++}$com solução de ace tato de cālcio a pH 7,0; pH em ăgua na relação 1:1. A percentagem de saturação de bases e a percentagem de saturação com alumínio foram calculadas pelas förmulas: $V \%=S+100 / T$ e $\% A 1=100 A 1 / A 1+S$. 
Nas repetições dos solos estudados foram medidas, altura total das árvores, com o hipsōmetro Blume-Leiss e o diāmetro a altu ra do peito, com a suta. O volume total foi calculado pela fórmula: $v t=h \times g \times f$. 0 fator de forma corresponde ao valor $0,57$.

os dados foram analisados utilizando-se correlação simples linear. Os teores dos elementos $\mathrm{Al}, \mathrm{Ca}, \mathrm{Mg}, \mathrm{P}$ e K dos solos, para efeitos de cálculos estatísticos, foram transformados em $\mathrm{kg} / \mathrm{ha}$.

RESULTADOS E DISCUSSÃO

Observa-se pelos resultados da Tabela I, nas condições des te estudo, que os teores de $\mathrm{Ca}+\mathrm{Mg}$ trocáveis apresentam correlação negativa e, quando separados, o Ca apresenta correlação mais eleva da e negativa com as variāveis do crescimento de Araucaria angustifolia do que o Mg. HOPPE (4) obteve relação semelhante entre a altura do minante e o teor de cālcio atē a profundidade de $50 \mathrm{~cm}$ e, para o magnésio nas duas profundidades estudadas.

TABELA I. COEFICIENTES DE CORRELAÇAOO SIMPLES LINEAR ENTRE VARIĀVEIS DO CRESCIMENTO DE Araucaria angustifolia E CARACTERISTICAS QUIMICAS DOS SOLOS ESTUDADOS, PARA A PROFUNDIDADE DE 0-20 CM.

\begin{tabular}{|c|c|c|c|c|c|c|}
\hline & $\bar{h}$ & $\bar{d}$ & $\mathrm{Ho}$ & ddom & $v t$ & IMA \\
\hline $\mathrm{Ca}^{++}+\mathrm{Mg}^{++}$ & $-0,927^{\star *}$ & $-0,861^{\star \star}$ & $-0,926^{\star \star}$ & $-0,945^{\star \star}$ & $-0,918^{\star \star}$ & $-0,918^{\star \star}$ \\
\hline $\mathrm{Ca}^{++}$ & $-0,944^{\star \star}$ & $-0,884^{\star \star}$ & $-0,937^{\star \star}$ & $-0,958^{\star \star}$ & $-0,924^{\star \star}$ & $-0,924^{\star \star}$ \\
\hline $\mathrm{Mg}^{++}$ & $-0,834^{\star *}$ & $-0,755^{\star}$ & $-0,851^{\star \star}$ & $-0,867^{\star *}$ & $-0,863^{\star \star}$ & $-0,863^{\star \star}$ \\
\hline $\mathrm{Al}^{+++}$ & $0,948^{\star \star}$ & $0,887^{* \star}$ & $0,975^{\star \star}$ & $0,980^{\star \star}$ & $0,986^{\star \star}$ & $0,986^{\star \star}$ \\
\hline S & $-0,939^{\star \star}$ & $-0,880^{\star *}$ & $-0,939^{\star \star}$ & $-0,947^{\star \star}$ & $-0,928^{\star *}$ & $-0,928^{\star *}$ \\
\hline$T$ & $-0,924^{* *}$ & $-0,886^{\star \star}$ & $-0,915^{\star \star}$ & $-0,923^{\star \star}$ & $-0,898^{\star \star}$ & $-0,898^{\star *}$ \\
\hline $\mathrm{H}^{+}+\mathrm{Al}^{+++}$ & $0,949^{\star *}$ & $0,888^{\star \star}$ & $0,973^{\star \star}$ & $0,981^{\star \star}$ & $0,983^{\star \star}$ & $0,983^{\star *}$ \\
\hline$\% \mathrm{~V}$ & $-0,952^{\star \star}$ & $-0,903^{\star \star}$ & $-0,956^{\star \star}$ & $-0,964^{\star \star}$ & $-0,937^{\star \star}$ & $-0,937^{\star \star}$ \\
\hline $100 \mathrm{~A} 1$ & $0,964^{\star \star}$ & $0,909^{\star \star}$ & $0,976^{\star \star}$ & $0,983^{\star \star}$ & $0,974^{\star \star}$ & $0,974^{\star \star}$ \\
\hline$A 1+S$ & & & & & & \\
\hline $\mathrm{pH} \mathrm{H}_{2} \mathrm{O} \quad 1: 1$ & $-0,845^{\star \star}$ & $-0,862^{\star \star}$ & $-0,839^{\star \star}$ & $-0,847^{\star \star}$ & $-0,783^{\star}$ & $-0,783^{\star}$ \\
\hline $\mathrm{P}$ & - & - & $-0,114$ & - & - & - \\
\hline K & $-0,568$ & $-0,566$ & $-0,584$ & $-0,513$ & $-0,557$ & $-0,097$ \\
\hline MO & 0,219 & 0,277 . & 0,219 & 0,205 & 0,259 & 0,004 \\
\hline
\end{tabular}

* Significância ao nível de $5 \%$.

* Significância ao nível de $1 \%$.

Pela observação de VAN GOOR (5) a Araucaria angustifolia necessita $2 \mathrm{me} / 100 \mathrm{~g}$ de solo de Ca + Mg trocāveis e, isto pode ser confirmado, para a profundidade de $0-20 \mathrm{~cm}$ (TABELA I). Por outro lado, o Re e HGP apresentam conteūdos superiores de Ca e Mg em rela ção ao LR(TABELA I), os crescimentos são lentos e, conseqdentemente, 
as produtividades obtidas nestes solos são inferiores (TABELA II).

TABELA II. VALORES DE ALTURA MEdiA $(\bar{h})$ E ALTURA DOMINANTE (Ho)*, DI METRO MEdio (d) E DiÂMETRO DOMinANTE (ddom), VOLUME TOTAL (Vt) E INCREMENTO MEDIO ANUAL (IMA) DE Araucaria angustifolia NOS SOLOS ESTUDADOS (FEVEREIRO, 1981).

\begin{tabular}{lcccccc}
\hline \hline Solos*** & $\frac{\hbar}{(\mathrm{m})}$ & $\frac{H_{0} *}{(\mathrm{~m})}$ & $\frac{\mathrm{d}}{(\mathrm{cm})}$ & $\frac{\mathrm{ddom}}{(\mathrm{cm})}$ & $\frac{V \mathrm{t}}{\left(\mathrm{m}^{3} / \mathrm{ha}\right)}$ & $\frac{\text { IMA }}{\left(\mathrm{m}^{3} / \mathrm{ha}\right)}$ \\
\hline LR & 16,90 & 19,27 & 24,23 & 34,67 & 416,67 & 16,17 \\
$\operatorname{Re}$ & 10,73 & 12,80 & 16,23 & 23,63 & 156,56 & 6,26 \\
HGP & 9,33 & 12,30 & 14,23 & 22,03 & 87,67 & 3,51 \\
\hline
\end{tabular}

* Definida pela média aritmética de 100 árvores de maior diâmetro por hectare.

** LR - Latosol Roxo, Re - Solo Litōlico Eutrōfico, HGP - Gley Pouco Hūmico.

Assim, apesar da associação obtida entre os teores de Ca e Mg dos solos com as variāveis do crescimento de Araucaria angustifolia, isto demonstra que, provavelmente, estes elementos, nas condições deste estudo, não são considerados os fatores limitantes.

Desta forma, os teores de Ca e Mg do LR são baixos (TABE LA III) e, possivelmente, neste caso, a abundāncia de micorrizas de tectadas nas camadas $\mathrm{F}$ e $\mathrm{H}$ e as que ocorrem em associação com Araucaria angustifolia até aproximadamente os primeiros $10 \mathrm{~cm}$ de solo mineral assumem grande importância no abastecimento de nutrientes. ANDRAE \& KRAPFENBAUER (1) e DE HOOGH (2) consideram importante a função de micorriza para nutrição de Araucaria angustifolia.

TABELA III. TEORES MEDIOS DE CARACTERISTICAS QUIMICAS DOS SOLOS ESTUdAdOS, PARA A PROFUNDIDADE $D E O-20 \mathrm{CM}$.

\begin{tabular}{|c|c|c|c|c|c|c|c|c|c|c|c|c|c|c|}
\hline \multirow[t]{3}{*}{ Solos* } & \multicolumn{8}{|c|}{ Teores trocāveis } & \multirow{3}{*}{$\begin{array}{l}v \\
\cdots\end{array}$} & \multirow{3}{*}{$\frac{100 \mathrm{~A} 1}{A 1+\mathrm{S}}$} & \multirow{3}{*}{$\begin{array}{l}\text { мо } \\
\ldots .\end{array}$} & \multicolumn{3}{|c|}{$\begin{array}{c}\text { Teores } \\
\text { disponiveis } \\
\end{array}$} \\
\hline & $\mathrm{Ca}^{++}$ & $\mathrm{Mg}^{++}$ & $\mathrm{k}^{+}$ & $\mathrm{Na}^{+}$ & $\mathrm{Al}^{+++}$ & $\mathrm{H}^{+}+\mathrm{Al}^{+++}$ & s & T & & & & & & $\mathrm{pH} \mathrm{H}_{2} \mathrm{O}$ \\
\hline & $\cdots$ & $\cdots$ & $\ldots \ldots \ldots$ & . me & $100 \mathrm{~g}$ & & & $\cdots \cdots$ & & & & $\mathrm{ppm}$ & $n \quad .$. & $1: 1$ \\
\hline$L R$ & 1,9 & 1,1 & 0,09 & 0,06 & 3,5 & 8,04 & 3,15 & 11,23 & 28 & 52 & 4,88 & 1,7 & 34 & 4,5 \\
\hline $\mathrm{Re}$ & 4,2 & 2,4 & 0,37 & 0,27 & 1,0 & 5,56 & 7,24 & 12,23 & 59 & 12 & 5,01 & 2,7 & 156 & 5,0 \\
\hline HGP & 6,2 & 3,3 & 0,33 & 0,19 & 0,7 & 6,87 & 9,91 & 16,78 & 59 & 6 & 4,87 & 1,3 & 106 & 5,1 \\
\hline
\end{tabular}

* LR - Latosol Roxo, Re - Solo litôlico Eutröfico, HGP - Gley Pouco Hümico.

Considerando-se que os ecossistemas florestais são comple xos e, levando-se em conta os fatores internos do sitio, como a ve getação que cresce em associação com Araucaria angustifolia, esta vegetação contribui para a composição de matéria orgānica do ambiente. 
Assim, supõe-se que a capacidade de decomposição dos restos vegetais do sub-bosque, bem como, da matéria orgânica do horizonte superfi cial do solo podem ocorrer de maneira rápida com a mineralização do humus e liberação de nutrientes. Isto porque as camadas L e F do La tosol Roxo estão constituídas, principalmente, por acículas e ga 1 hos de Araucaria angustiforia.

Alēm disso, os nutrientes contidos na serrapilheira do LR, os tecidos orgânicos mortos e também os nutrientes de restos de raí zes subterrâneas contribuem para o reservatōrio e, para a circula ção de nutrientes. ANDRAE \& KRAPFENBAUER (1) encontraram quantida des apreciāveis de elementos fixados na serrapilheira. Desta forma, devido à alta temperatura e precipitação bem distribuída, os elemen tos podem ser ciclados, possivelmente a uma velocidade rápida de ma neira que não ocorre acumulação no solo, principalmente, no LR.

Por outro lado, a metodologia empregada para a extração dos elementos do solo, provavelmente, não seja a mais recomendāvel para solos sob florestas, pois DE HOOGH (2) diz que os resultados obtidos pela digestão total do solo correlacionaram-se melhor do que os te $\underline{0}$ res trocāveis. Também, deve-se considerar o ciclo de rotação de po voamentos florestais e, possivelmente, os teores trocáveis que ocor rem no momento, podem com o decorrer do tempo estar disponíveis pa ra as plantas.

obteve-se correlação positiva entre o alumínio trocāvel e as variāveis do crescimento de Araucaria angustifolia. Semelhante relaçāo ocorre com a percentagem de saturação de alumínio, (TABELA I). Entretanto, não significa que o alumínio seja necessārio para esta espécie e o efeito deste elemento pode estar relacionado indireta mente às características pedogenéticas do LR, uma vez que os melho res crescimentos de Araucaria angustifolia encontram-se neste solo (TABELA II). Provavelmente, o efeito negativo do nivel de Al do LRem plan tas adultas de Aracaria angustifolia seja superado pelo fato deste solo apresentar boas condições físicas para o crescimento.

A soma de bases, bem como, a percentagem de saturação de bases, mostraram correlação negativa com as variāveis do crescimen to de Araucaria angustifolia (TABELA I). DE HOOGH \& DIETRICH (3)obti veram correlação positiva entre o indice de sītio e a percentagem de saturação de bases. A relação obtida, possivelmente, pode ser ex plicada pelo fato destas características estarem relacionadas com 0 conteūdo de $\mathrm{Ca}$ e $\mathrm{Mg}$ do solo.

A capacidade de troca apresentou correlação negativa com as variāveis do crescimento de Araucaria angustifolia (TABELA I). Es ta relação pode ser interpretada como uma relação casual, uma vez que o conteūdo de matēria orgānica pode contribuir em grande parte 
no complexo de absorção, principalmente, no LR.

A acidez de troca e pH em āgua na relação 1:1 dos solos estudados, correlacionaram-se negativamente com as variáveis do cres cimento de Araucaria angustifolia (TABELA I).

Não foram observadas correlações entre os elementos P e $K$ disponíveis, nem para o conteūdo de matēria orgānica dos solos e as variáveis do crescimento de Araucaria angustifolia, (TABELA I).

Uma diminuição nos teores de $\mathrm{Ca}+\mathrm{Mg}$ trocāveis, $\mathrm{pH}$, acidez de troca, capacidade de troca, soma de bases, percentagem de satura ção de bases e um maior conteüdo de alumínio e consequlentemente, maior percentagem de saturação de alumínio, associam-se aos melhores cres cimentos e produtividade de Araucaria angustifolia, (TABELA II).

Entretanto, as características químicas, nas condições des te estudo, não representam os melhores parâmetros do solo, para ex plicar a variação do crescimento de Araucaria angustifolia e, muitas correlações obtidas podem estar relacionadas às caracterīsticas pe dogenēticas do solo.

\section{CONCLUSOES}

Considerando-se os resultados obtidos, chegou-se a conclu são, nas condições deste estudo, que as características quỉmicas do solo não serviram como parāmetros, para explicar a variação do cres cimento de Araucaria angustifolia.

LITERATURA CITADA

1. ANDRAE, F.H. \& KRAPFEnBAUER, A. - Inventur einer 17 . jăhrigen Araukarienaufforstung in Passo Fundo, Rio Grande do Sul, Bra silien. Centralbz. fllr das Gesamte Forstwesen, 93(4):203-30, 1976 .

2. DE HOOGH, R.J. - Site - nutrition - growth relationships of Araucaria angustifolia (Bert.) 0. Ktze. in Southern Brasiz. Freiburg. 161 p. Tese Ph. D. 1981.

3. - \& DIETRICH, A.B. - Avaliação de sítio para Araucaria angustifolia (Bert.) 0. Ktze. em povoamentos artificiais. Bra sil Elorestal, (37):19-71, 1979.

4. HOPPE, J.M. - Relagões entre dados analiticos do solo, análise foliar e dados de incremento de Araucaria angustifolia (Bert.) 0. Ktze., na FLONA de Passo Fundo, RS. Curitiba. 90 p. Dis sertação (Mestr. Engå.. Fta1.). UFPR, Curitiba, 1980.

5. VAN GOOR, C.P. - Classificação da capacidade da terra em relação ao reflorestamento com Pinus elziottii Eng. var. elliottii e Araucaria angustifolia (Bert.) 0. Ktze., no Estado de São Paula. Silvicultura em sä́o Paulo, (4): 349-66, 1965/66. 
6. VetToRi, L. - Métodos de análise de solo. Rio de Janeiro. Minis s tério da Agricultura. Divisão de Pedologia e Fertilidade do Solo. 1969. 24p. (Boletim Técnico, 7).

Recebido em maio, 1983; aceito em agosto, 1983. 
